\title{
SETI AS A SCIENCE
}

\author{
David W. Swift \\ University of Hawaii, Dept of Sociology \\ Honolulu, HI 96821 USA
}

\begin{abstract}
$\underline{\text { Summary }}$
Interviews with pioneers in the search of extraterrestrial intelligence clarify questions about the nature of the search.' Despite its 'far out' objective, it shares characteristics with other, more conventional activities which are considered to be "science".

However, SETI does face greater difficulties than many other new fields, as suggested by questions such as the following:

Does the phenomenon exist?

When can results be expected?

What useful purposes will the project serve?
\end{abstract}

\section{Reference}

1. Swift, D. W., 1990: SETI Pioneers, University of Arizona Press, Tucson, AZ.

\section{$\underline{\text { Discussion }}$}

N. EVANS: I think that the main reason SETI may not be science is that it will be very difficult to falsify the hypothesis that extraterrestrial intelligence exists. Even if all our planned searches fail, I doubt that most of us would conclude that no ETI exists, and we would be even less likely to conclude that no extraterrestrial life (e.g., bacteria) exists. Rather than science, I think SETI is something more important: a voyage of discovery.

D. SWIFT: Are Discovery and Science mutually exclusive? 